\title{
The prognostic importance of parotid involvement by head and neck squamous cell carcinoma - Case report ${ }^{*}$
}

\author{
Bruna Melhoranse Gouveia ${ }^{1}$ \\ Leonardo Hoehl Carneiro ${ }^{1}$ \\ Nurimar Conceição Fernandes ${ }^{1}$
}

\author{
Maria Helena de Magalhães Barbosa ${ }^{1}$ \\ Luzia Abrao El Hadj ${ }^{1}$
}

DOI: http:/ /dx.doi.org/10.1590/abd1806-4841.20163995

\begin{abstract}
Squamous cell carcinoma (SCC) is the second-most common malignant cutaneous cancer, with $60 \%$ occurring in the head and neck region. Metastases are uncommon and imply a more conservative prognosis. This report describes a case of parotid-invasive, facial squamous cell carcinoma, highlighting the importance of its prognostic and therapeutic management. The patient is an 81-year-old female, exhibiting extensive tumoral lesions in the pre-auricular region, affecting the parotid parenchyma and implying the metastatic involvement of the intra-parotid lymph node. Parotid involvement caused by SCC in specificity tumors is discussed herein. Parotid invasion is currently recognized as an isolated variable. It affects survival rates and determines certain changes in case management, such as the broadening of resection areas and adjuvant radiotherapy.
\end{abstract}

Keywords: Head and neck neoplasms; Skin neoplasms; Parotid Neoplasms; Carcinoma, squamous cell; Parotid region; Prognosis

\section{INTRODUCTION}

Squamous cell carcinoma (SCC) is the second-most common cutaneous malignancy, with $60 \%$ occurring in the head and neck region. ${ }^{1}$ The rate of head and neck SCC metastases is estimated to occur in less than $5 \%$ of patients. In such cases, parotid metastases are around $3 \%$ and indicate aggressive local disease, as well as more severe prognosis. ${ }^{2}$ In this case, the importance of parotid involvement is reported as an isolated prognostic factor and a therapy guide.

\section{CASE REPORT}

An 81-year-old, female patient had a tumor in the left pre-auricular region, with 3 months of evolution. Upon physical examination, the tumor was 2.5 $\mathrm{cm}$ in diameter, well-delimited, with an ulcerated center exhibiting yellowish exudates and a hematic crust (Figure 1). No lymph nodes were detected in the physical exam. Head and neck computer tomography detected a lesion in the skin that reached the parotid parenchyma (Figure 2). Surgical excision was performed with cutaneous margins of $5 \mathrm{~mm}$, in addition to a parotidectomy and lymphadenectomy of the level II cervical nodes (Figure 3). Histopathology revealed SCC with margins free and a tumor of $0.5 \mathrm{~cm}$ in the intraparotid nodes (Figures 4, 5 and 6). Level II lymph nodes were tumor-free. There was no perineural invasion. Thus, the patient was classed as P1 N0, following the $\mathrm{O}^{\prime}$ Brien classification, according to which " $\mathrm{P}$ " corresponds to "parotid involvement" and " $\mathrm{N}$ " to "Neck disease". ${ }^{3}$ As evolution, the patient presented a temporary, peripheral, facial palsy on her left side for two months. She has been kept in follow-up, and has completely recovered movement. Furthermore, she refused to undergo radiotherapy and did not show any metastatic involvement three-year post-surgery. 


\section{DISCUSSION}

Parotid nodes receive lymphatic drainage from a large area because this gland is the first to form and the last to encapsulate during the embrionary process. Thus, parotid nodes have long been recognized as potential sites for lymphatic spread of tumors but their importance in the prognosis of SCC in the head and neck region has emerged relatively recently. ${ }^{3}$ The current AJCC TNM staging system does not account for the importance of parotid involvement. In TNM staging, all patients with parotid and/or neck nodal metastasis are classified as N1. ${ }^{4}$ Thus, O'Brien proposed a new classification to distinguish between parotid node involvement (" $\mathrm{P}$ ") and neck nodal disease $(" N$ "). This classification considers that the higher the

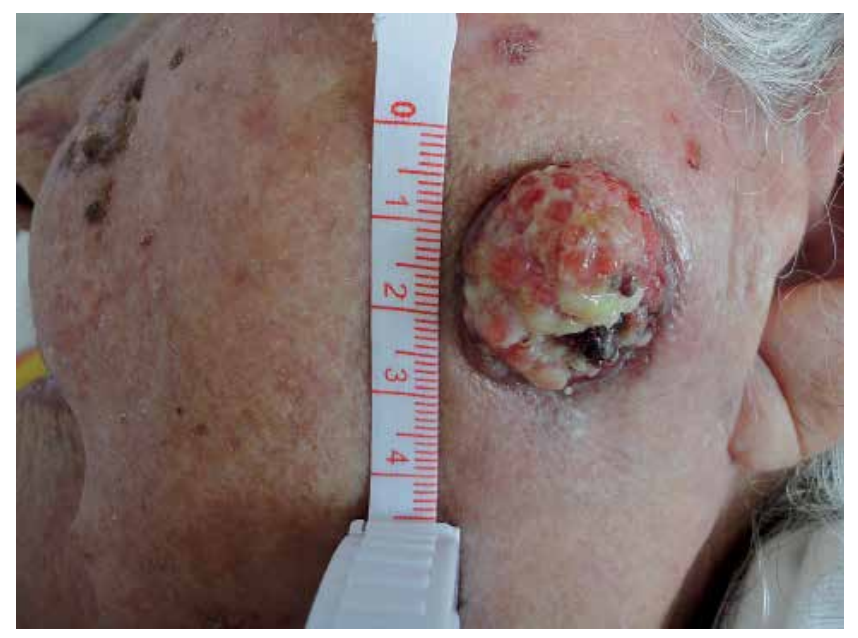

Figure 1: Clinical features of tumor in left pre-auricular regions

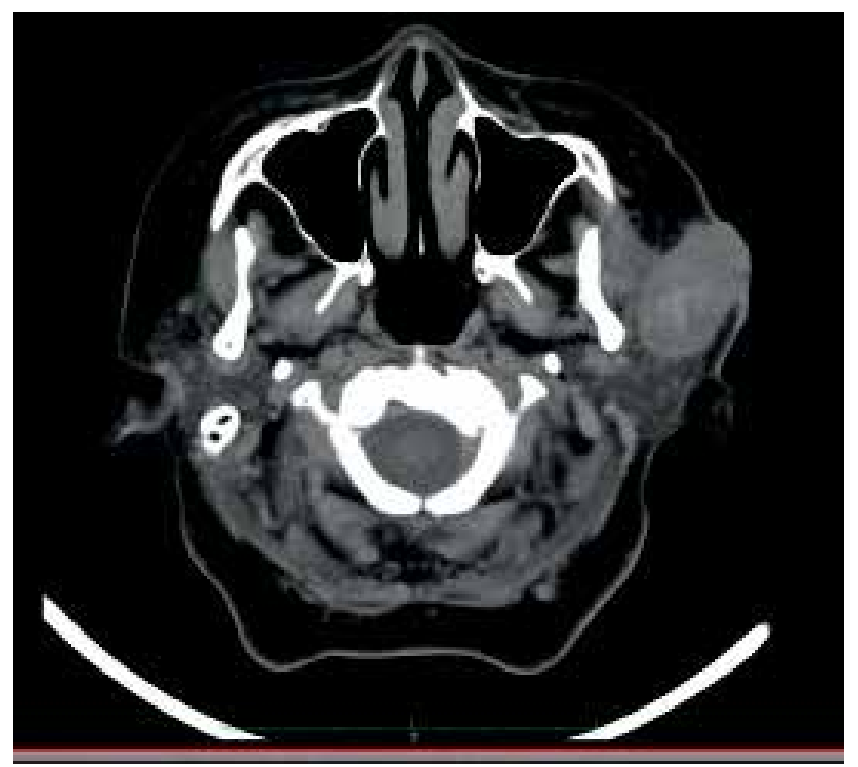

Figure 2: Head and neck computer tomography detected lesion extent and depth

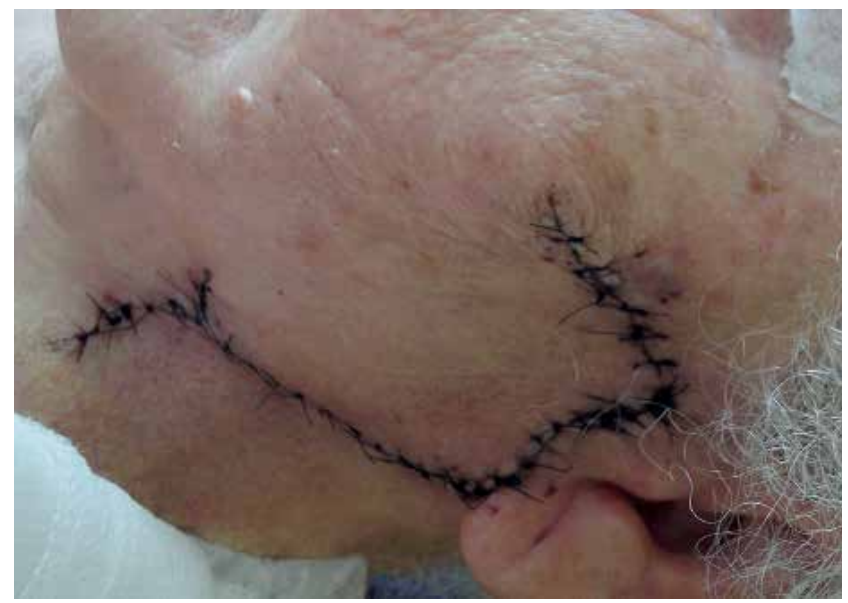

FIGURE 3: Post-surgery aspect

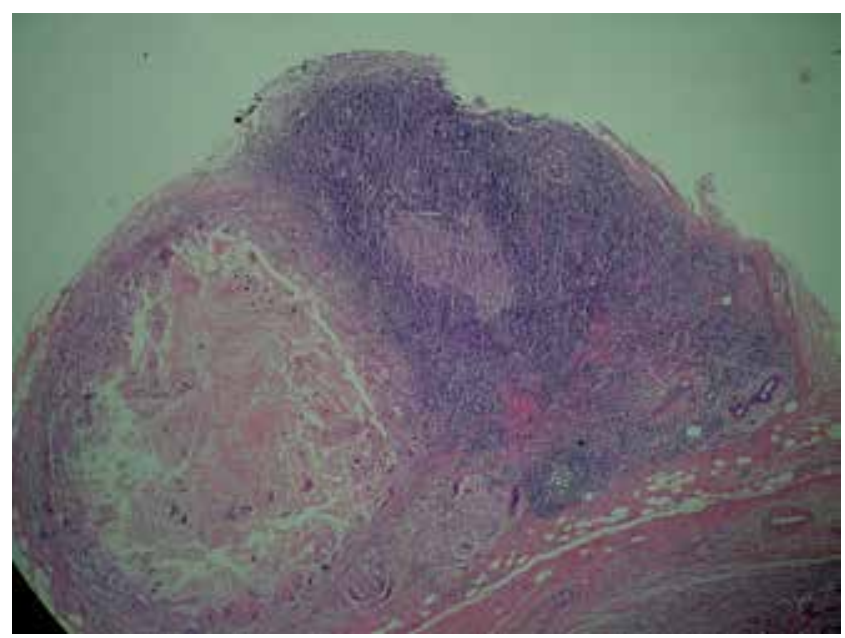

Figure 4: Histopathological study: Intraparotid lymphonode exibiting metastatic squamous cell carcinoma with large central necrotic area (HE, 100X)

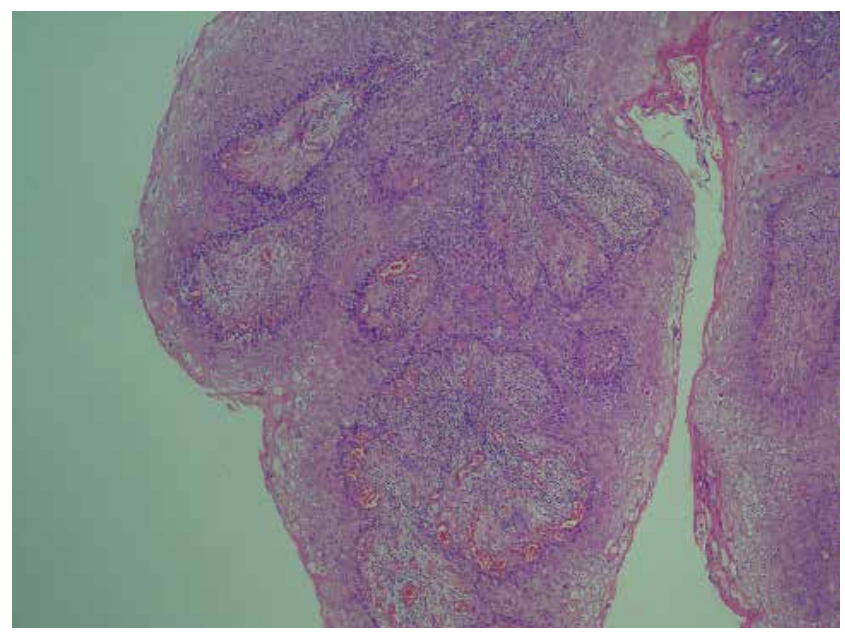

FIGURE 5: Histopathological study: Squamous cell carcinoma with papillary architecture (HE, 100X) 


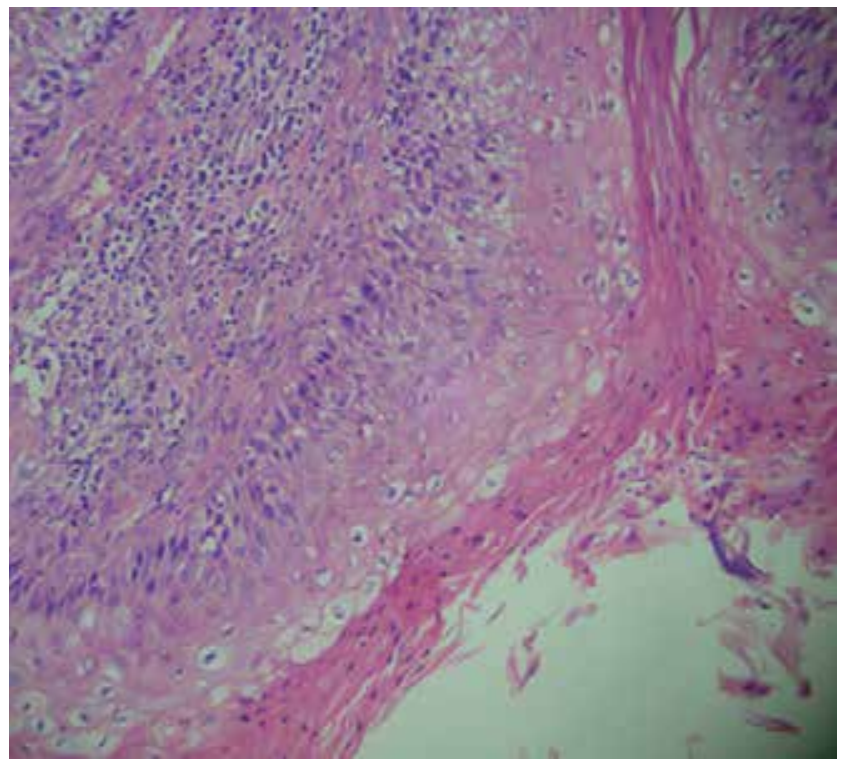

FIGURE 6: Histopathological study: Neoplastic cells with clear squamous differentiation (HE, 400X)

parotid involvement, the lower the survival index. For early P stages (P0 - no parotid involvement - or P1 - metastatic parotid node of up to $3 \mathrm{~cm}$ ), the survival index is about $82 \%$. In contrast, at later stages (P2 metastatic parotid node of $3-6 \mathrm{~cm}$ or multiple nodules; or P3 - metastatic parotid node $>6 \mathrm{~cm}$ or involvement of facial nerve or skull base), the survival index is $69 \%$. Prospective multicenter studies are warranted to ar- rive at a staging system that better prognosticates for cutaneous SCC but one criterion is well-known: parotid involvement as a prognostic factor in itself. ${ }^{1,2}$ There are many risk factors that increase significantly the risk of parotid nodal metastasis: tumor sizes of over $2 \mathrm{~cm}$ in diameter, invasion depths above $5 \mathrm{~mm}$ in thickness, proximity to the parotid gland and advanced age. ${ }^{5}$ For these patients, some authors have proposed dual modality treatment with surgery and adjuvant radiotherapy (RT) but there is no clear consensus yet. ${ }^{6}$ Several studies have demonstrated that dual-modality treatment is necessary for head and neck metastatic cutaneous SCC with adverse histological factors. These factors are: extracapsular spread, close or incomplete margins, multiple nodal involvement and perineural spread. ${ }^{4}$ European guidelines suggest RT should be used only in a minority of cases and that the indication of lymphadenectomy should be guided by clinical examination and loco-regional ultrasound. ${ }^{7}$ In this case, the patient refused to undergo RT but no consensus exists yet regarding its indication. She presented no adverse histological factors warranting RT and no neck involvement, and was thus kept in follow-up. Hence, more studies are necessary to define criteria for RT and lymphadenectomy in SCC, as well as prospective studies to identify more prognostic factors and their importance in parotid involvement.]

\author{
MAILING ADDRESS: \\ Bruna Melhoranse Gouveia \\ Rua Rodolpho Paulo Rocco, 255 \\ Cidade Universitária \\ Ilha do Fundão \\ 21941-913 - Rio de Janeiro - RJ \\ Brazil \\ E-mail: brunamelhoranse@gmail.com
}

5. D'Souza J, Clark J. Management of the neck in metastatic cutaneous squamous cell carcinoma of the head and neck. Curr Opin Otolaryngol Head Neck Surg. 2011;19:99-105.

6. Bumpous J. Metastatic cutaneous squamous cell carcinoma to the parotid and cervical lymph nodes: treatment and outcomes. Curr Opin Otolaryngol Head Neck Surg. 2009;17:122-5.

7. Bonerandi JJ, Beauvillain C, Caquant L, Chassagne JF, Chaussade V, Clavère $P$ et al. Guidelines for the diagnosis and treatment of cutaneous squamous cell carcinoma and precursor lesions. J Eur Acad Dermatol Venereol. 2011;25:1-51.

How to cite this article: Melhoranse BG, Barbosa MHM, Carneiro LH, El Hadj LA, Fernandes NC. The prognostic importance of parotid envolvement by head and neck squamous cell carcinoma - Case report. An Bras Dermatol. 2016;91(3):351-3. 